Monat krank gemeldet, im Mittel knapp sechs Tage. Ohne psychische Störung war nur ein Zehntel krank, und dies mit im Schnitt nur 3,5 Tagen. Die DEGS-Zahlen liegen aber noch immer weit über den administrativen Daten zu psychisch bedingten Fehlzeiten von Krankenkassen. Wenn also in regelmäßigen Abständen gemeldet wird, dass immer mehr Menschen wegen psychischer Erkrankungen am Arbeitsplatz fehlen, liege das lediglich daran, dass die Fehlzeiten spezifischer als früher auf psy- chische Diagnosen bezogen werden. „Es gibt aber keine Zunahme psychischer Störungen“, fasste Professor Hans-Ulrich Wittchen, Technische Universität Dresden, zusammen. Darauf deuteten Vergleiche mit dem Bundesgesundheitssurvey von 1998 ebenso wie europaweite Analysen. Thomas Müller, Springer Medizin

Symposium „Das Modul Mental Health in der Studie zur Gesundheit Erwachsener in Deutschland (DEGS)“, DGPPN-Kongress, Berlin, 23.11.2012

\title{
Schizophrenieprävention
}

\section{Psychose lässt sich bei jedem Zweiten verzögern oder verhindern}

\begin{abstract}
Psychotherapie, Medikamente oder gar Fischöl? Noch ist unklar, womit sich eine Psychose am besten verhindern lässt. Klar ist, dass unterschiedliche Therapien greifen und die Psychoserate bei gefährdeten Personen halbieren.
\end{abstract}

„Es gibt eine Menge Daten, die zeigen, dass Psychoseprävention funktioniert. Jetzt ist es an der Zeit, die Erkenntnisse in die Vorsorge zu übernehmen", forderte Professor Andreas Bechdolf, Vivantes Klinikum Berlin. Er erinnerte daran, dass nach Ergebnissen einer aktuellen Metaanalyse mit über 2.500 psychosegefährdeten Teilnehmern $32 \%$ der Betroffenen ohne Behandlung innerhalb von drei Jahres eine manifeste klinische Erkrankung entwickeln. Solche Menschen sind meist schon vier bis fünf Jahre vor einer ersten Episode auffällig und haben kurze, selbstlimitierende psychotische Symptome, wie etwa eigentümliche Vorstellungen, Sprech- und Denkweisen oder paranoide Ideen, wobei ein Realitätsbezug noch vorhanden ist. Für ein hohes Risiko sprechen auch eine familiäre Belastung und ein Funktionsabbau, etwa sozialer Rückzug oder kognitive Defizite. Wenn solche Personen Hilfe suchen, dann häufig wegen ihrer Funktionseinschränkungen, Behinderung, Ängste und Depressionen. „Hier müssen wir sie unterstützen, damit sie überhaupt zu uns in ein Früherkennungszentrum kommen", sagte Bechdolf. Erst im zweiten Schritt gehe es darum, die Patienten für eine Intervention zu gewinnen, um eine psychotische Episode zu verhindern. Je früher es aber gelinge, psychosegefährdete Personen zu behandeln, umso besser ist die Prognose.

\section{Verhaltenstherapie und Medikamente - beides wirkt}

In den vergangenen zehn Jahren wurde gut ein Dutzend randomisiert-kontrollierter Studien veröffentlicht, die den Nutzen einer Intervention im Prodromalstadium belegen. Meist wurden dabei eine kognitive Verhaltenstherapie (KVT), atypische Neuroleptika sowie eine Kombination aus beidem gegen Placebo und ein unspezifisches klinisches Management geprüft. In fast allen Studien war die spezifische Intervention einer unspezifischen Unterstützung signifikant überlegen: Mit Psychotherapie oder Medikamenten entwickelten nur etwa 5-15\% der Teilnehmer innerhalb eines Jahres eine Psychose, in den Kontrollgrup- pen dagegen $20-35 \%$. In der Regel konnte die spezifische Intervention die Psychoserate halbieren bis vierteln. Noch ist allerdings nicht klar, welche Form der Intervention am meisten bringt. In den Studien waren sowohl KVT als auch Medikamente der unspezifischen Intervention deutlich überlegen, die Kombination der beiden Strategien brachte aber meist keinen zusätzlichen Nutzen. Bechdolf plädiert daher für den Beginn mit einer gut verträglichen psychosozialen Intervention. Erst bei Nichtansprechen oder wenn weitere Risikofaktoren wie Suizidalität hinzukommen, sollte man zu Neuroleptika greifen.

Für interessant hält der Psychiater auch Präventionsstudien mit Omega-3-Fettsäuren. In einer Studie mit 81 Patienten entwickelten nur $5 \%$ der Hochrisikopersonen mit einer Omega3-Therapie innerhalb eines Jahres eine Psychose, ohne waren es $25 \%$. Die Ergebnisse werden nun in einer neuen Studie überprüft. Als Wirkmechanismus vermuten Forscher neuroprotektive Eigenschaften der Fettsäuren.

Aufgrund des Nutzen präventiver Therapien bei Schizophrenie schlägt Bechdolf vor, ein Prodromalstadium in den ICD-10 aufzunehmen. Dann ließen sich psychosegefährdete Personen besser versorgen. Eine Aufnahme in den ICD-10 würde es auch erleichtern, Leitlinien für eine strukturierte Behandlung psychosegefährdeter Menschen zu entwickeln.

Neue Hinweise zum Nutzen der Prävention könnte die noch laufende deutsche PREVENT-Studie liefern. In der dreiarmigen multizentrischen Studie erhalten 300 Teilnehmer mit hohem Psychoserisiko entweder eine KVT, eine Therapie mit niedrig dosiertem Aripiprazol oder Placebo. Die Analyse der Basisdaten zeigt gewisse geschlechtsspezifische Unterschiede: Männer sind häufiger arbeitslos, drogen- oder alkoholabhängig als Frauen, zeigen eine stärkere Negativsymptomatik und ein schlechteres Funktionsniveau. Ein Großteil dieser Unterschiede lasse sich darauf zurückführen, dass Männer länger warten, bis sie Hilfe suchen und die Erkrankung daher bei ihnen im Schnitt schon weiter fortgeschritten ist, sagte Diplompsychologe Liz Rietschel vom Uniklinikum Hamburg-Eppendorf. Thomas Müller, Springer Medizin

Symposium „Auf dem Weg zur präventiven Psychiatrie“, Symposium „PREVENT: Erste Ergebnisse eines integrativen Studienverbundes bei Personen mit erhöhtem Psychoserisiko", DGPPN-Kongress, Berlin, 22.11.2012 